\title{
The Research of Eiderdown Quantitative Conveying and Automatic Weighing Method Based on PLC Control
}

\author{
Huichao Shang ${ }^{1,}$, Mengzhen Duan ${ }^{1, b}$, Xiaowei Duan ${ }^{1, c}$ and \\ Hongbin Zhang ${ }^{2, d}$ \\ ${ }^{1}$ School of Mechatronics Engineering, Zhongyuan University of Technology, Zhengzhou 451191, \\ ${ }^{2}$ Henan Xinya Clothing co., Ltd., Hebi 456750, China. \\ ash_hc@126.com,,b1551778622@qq.com, ${ }^{\text {a } 840617049 @ q q . c o m,, 283720621 @ q q . c o m ~}$
}

Keywords: Automatic Weighing, Pneumatic Conveying, PLC Control, PLC Dual Airflow Control.

\begin{abstract}
The key problem during the process of dynamic quantitative weighing for eiderdown outwear lies in the optimization and balance of weighing precision and weighing speed, regarding which a kind of method of quantitative conveying and automatic weighing was designed in this paper. Using pneumatic conveying method of controlled by PLC, variable airflow pressure was controlled by PLC through dual to adjust the conveying speed of down feather by time interval, a weighing sensor was applied to make a feedback on the real-time weighing data, and the timely changing-over of air channel was controlled by PLC. The weighing experiment was conducted when the combination of different weighing values and different pressure values and different percentages, least squares fit was conducted to gain the function relationship between air pressure and time and find out the optimal air pressure value available under quantitative weighing; in addition, PLC programming was applied to realize the control automatic switch over of air flow passage, finally rapidly and precisely weighing down feather.
\end{abstract}

\section{Introduction}

During the productive process of eiderdown outwear, the weight of eiderdown needs to be accurately weighed and then get it fill in the clothes exactly. China is the production super-country for eiderdown. But now the weighing of eiderdown also mainly depends on the traditional manual operation [1]. The traditional operation has a low degree of accuracy and production efficiency, and it's difficult for weight of eiderdown to be controlled in the accuracy range [2][3]. According to the demands of enterprise, this article introduces a new dynamic and quantitative weighing system. It takes the FATEK FBs-60MAT2-AC type PLC as system core to design the software and hardware, and develops series of weighing experiments to realize the quick and accuracy weighing for eiderdown.

\section{Research Status of Eiderdown Weighting at Home and Broad}

In our country, there are many patents in the research filed of eiderdown weighing devices, but many of them are made improvement on the mechanical parts. For example, the "weighing box" of Changshu Bealead Auto Machinery[3], it is a new weighing box for eiderdown including vertical box and weighing box. The structure is simple and it is convenient for weighing sensor. The "eiderdown weighing box used for filling machine for eiderdown" [4] produced by the Suzhou Biyangte Garment Equipment Co., Ltd. has provided a new equipment that it can avoid the transformation of weighing box that when bring the eiderdown in the weighing box, it can get the air with eiderdown discharged into outside and increase the settling rate of eiderdown to increase the speed and accuracy of weighing. As for the "anti-static weighing box for eiderdown" [5] produced by Suzhou Biyangte Garment Equipment Co., Ltd., it connects the introductory pipe or transfer pipe with one side of the static-erasure, the other end of static-erasure connects the ground. It is benefit to eliminate the static to 
guarantee the accuracy of weighing and reduce the security risk brought by the static. As for "accurate weighting box" [6] produced by Suzhou Transparent Electric Technology Co., Ltd., when it transports the eiderdown, the airflow enters into the frame screen from intake-tube, then rushes out from the frame screen and the relative side wall of intake-tube. Therefore it can avoid the waggle of weighing box because of the shocking box wall for gas to guarantee the accuracy of weighing. As for the "weighing box used for filling machine of eiderdown with buffering function" [7] produced by the Changshu Bealead Auto Machinery, it can additional set butter tank to reduce the buffer time of weighing.

In terms of weighing control, in the 1990 s Zhao Yanwei and Wang Wanliang etc adopted the weighing sensor in form of electric resistance strain gage. The measuring circuit adopts four foil-type resistance strain gauges to constitute the Wheatstone bridge. It proposed the filtering prediction algorithm based on Chebyshev approximation and intelligent control rules and therefore it can forecast and control the weight of eiderdown in the weighing box at any time. The fundamental is that it can confirm the forecasting steps according to the current eiderdown-in rate to make corresponding weighing forecast and change the gas circuit to realize the weight control according to the forecasting information[2][8 9]. Qian Qiao etc proposed the "automatic eiderdown-filling equipment based on the ARM7TDMI nucleus", it adopts the embedded control system HMS30C7202 to realize the automatic and intelligent weighing control and uses corresponding theories of fuzzy control algorithm to design the fuzzy controller. The eiderdown weight of output is the input weight of fuzzy algorithm and the rate of negative-pressure fan and opposite time of filling eiderdown is regarded as the output weight to control and realize the algorithm [10 11] through program in controller. It can be known from the "design and research on filling machine for eiderdown based on PLC control system"[3] and "design and research on automatic filling machine for eiderdown based on PLC"[12], during the PLC control it adopts the matched weighing module with weighing sensor and uses the weighing sensor to show the real-time weight data. And it uses the PLC closed-loop control algorithm to adjust the output rate of frequency converter in time to get the goal for switchover of control rate for different periods when weighing.

\section{Weighing Flow of Eiderdown}

\subsection{Basic Structure of Weighing.}

The basic structures of weighing system are shown as Figure 1, it is mainly constituted by the eiderdown-storage box, weighing box, material valve, air amplifier, air compressor, weighing sensor, PLC and touch screen etc. The eiderdown-storage box and weighing box are connected by the interflow material pipes and gas circuits. The eiderdown-storage is used to storage the stand-by eiderdown. In the bucket there is equipped with the stirring motor, with continuous stirring, it can loose and scatter the eiderdown adequately. So that when the eiderdown is fluffy it can spread in the eiderdown-storage evenly to be the floating conditions to avoid the stack of eiderdown for complete the transportation of eiderdown for weighing. The bottom of weighing box is equipped with a weighing sensor, for the weighing of this system is not more than $300 \mathrm{~g}$, so it adopts the S406C3MR/600g weighing sensor produced by Shenzhen Sen Si Er Technology Co., Ltd. to guarantee the accuracy of weighing. The weighing grade is $\mathrm{C} 2$, and the sensitivity is high and structure is simple. The Q611F pneumatic ball value has a small volume, simple configuration and big flow capacity, especially it is fit for the occasions with thick character, grain and fiber. It is widely used for the industrial automatic control system of light industry, chemical industry, teaching and scientific research equipments etc industries. Therefore it is adopted to be the material valve to control the on-off for eiderdown from eiderdown-storage bucket to weighing box. 


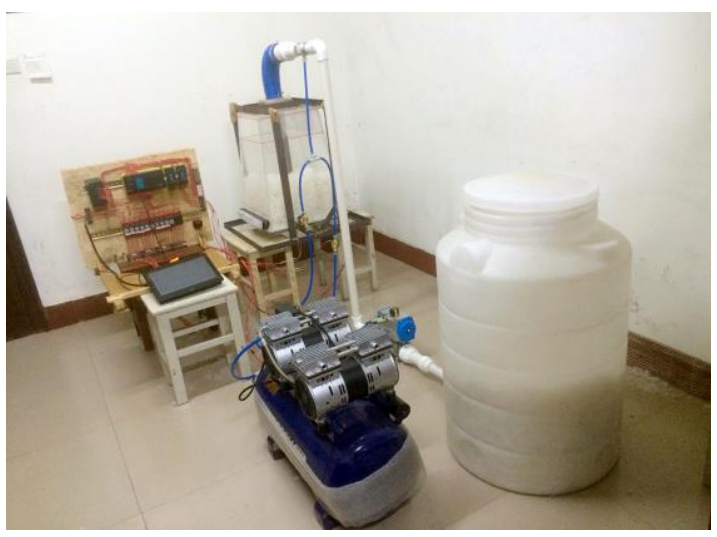

Fig.1 Basic structure of weighing system
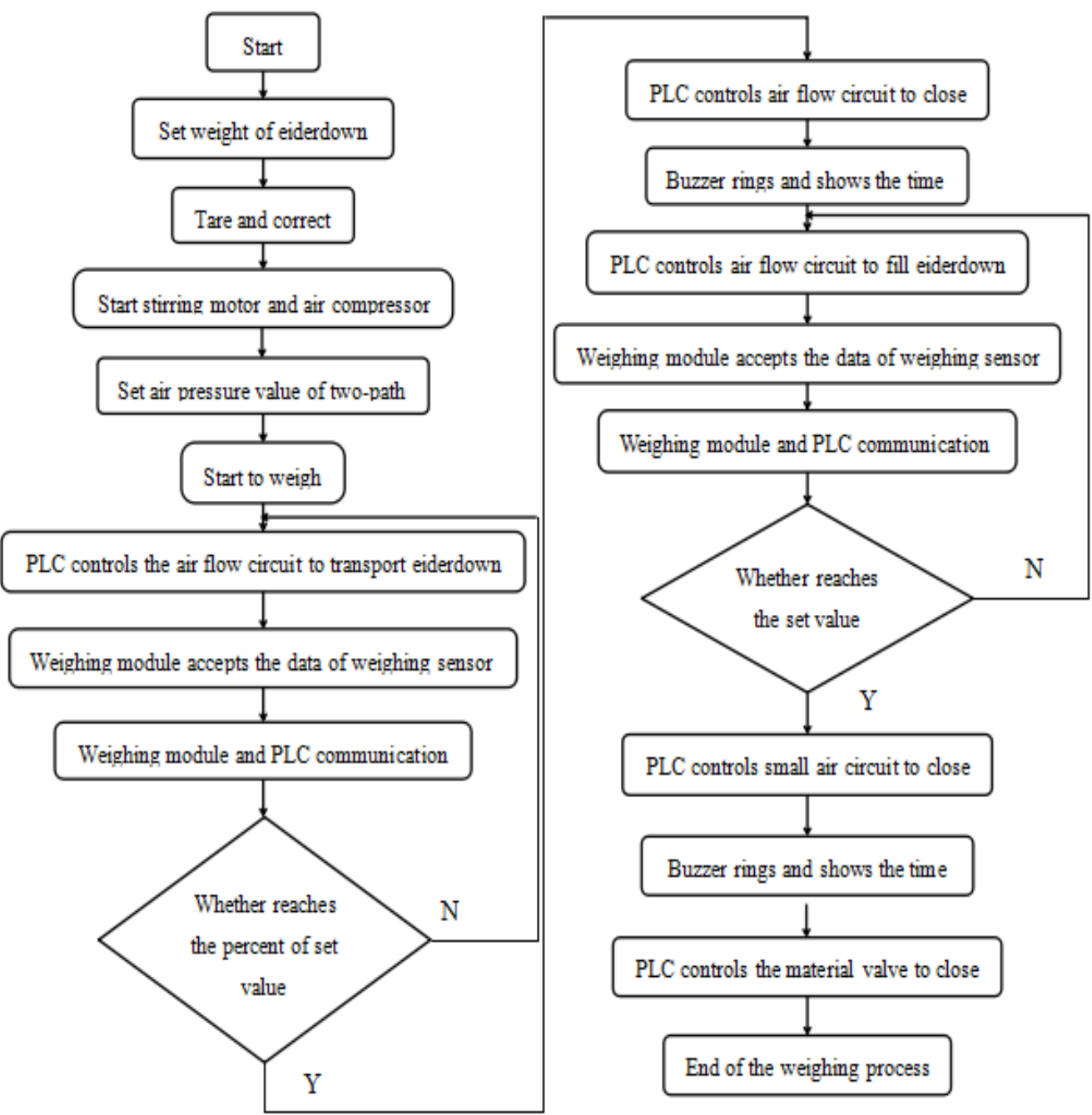

Fig.2 Route chart for weighing system

\subsection{Basic Workflow of Weighing.}

Before weighing, it is required to set the value needed to weigh on the touch screen and complete the tare and check. When weighing the worker is required to place enough eiderdowns on the eiderdown-storage box and then turn on the stirring motor. Through the movement of agitator arm the eiderdown will be fully scattered to complete the process of preparing material. Start the air compressor, the compressed air will produce negative pressure through the air amplifier. The eiderdown is imported the weighing box through the running pipe using the principle of negative pressure. And then it is required to manually set up the air pressure of two-path. The open of material valve and gas flow circuit is controlled by PLC, and this time eiderdown is being transporting from 
eiderdown-storage box to weighing box. The weighing sensor on bottom of weighing box can make the real-time collection and transport the weight information to weighing module. PLC obtains the weight data through internal wiring and communication of weighing module and judge whether the eiderdown can reach the percent of set value. After reaching the percent of set value, the flashlight buzzer will ring for 2 seconds, after 2 seconds PLC controls the small air flow circuit to disconnect. At the same time inner timer of PLC records the elapsed time for completing this process and shows it on the touch screen. After the weight of eiderdown reaches set value for 0.01 second, PLC controls the break of small air flow circuit and shows the elapsed time for completing this process. After 2 seconds for break of small air flow circuit, PLC controls the close of material valve. This is the whole weighing process.

\subsection{Weighing Control Process.}

The efficiency and stability of eiderdown transporting directly influences the efficiency and accuracy of weighing. On the dynamic quantitative weighing process of eiderdown, if the set value of air pressure is bigger, it can guarantee the transportation speed of eiderdown, but the big airflow will influence the weighing box and bottom of weighing sensor to influence the weighing precision. In addition the fluid characteristic of transportation flow and hysteresis quality of weighing data will cause the comparatively accurate weighing result which is difficult to be obtained. On the contrary if the set value of air pressure is small, it can obviously reduce the influence of airflow pressure to weighing box and bottom of weighing sensor. And it can timely close the airflow when the weight of eiderdown reaches the set value, so that it can confirm the veracity of weighing result. But this will greatly decrease the transportation speed and weighing efficiency. Aiming at this situation, this article made design for the weighing process and set two airflow controls to the transporting process of eiderdown. The air flow circuit can reach the presupposed percent of eiderdown weight in short time and decrease the weighing time. On the contrary the small airflow circuit can confirm the weighing precision of eiderdown. On the process of weighing PLC program can realize the switchover of big and small airflow circuit, so that it can complete the weighing process rapidly and accurately.

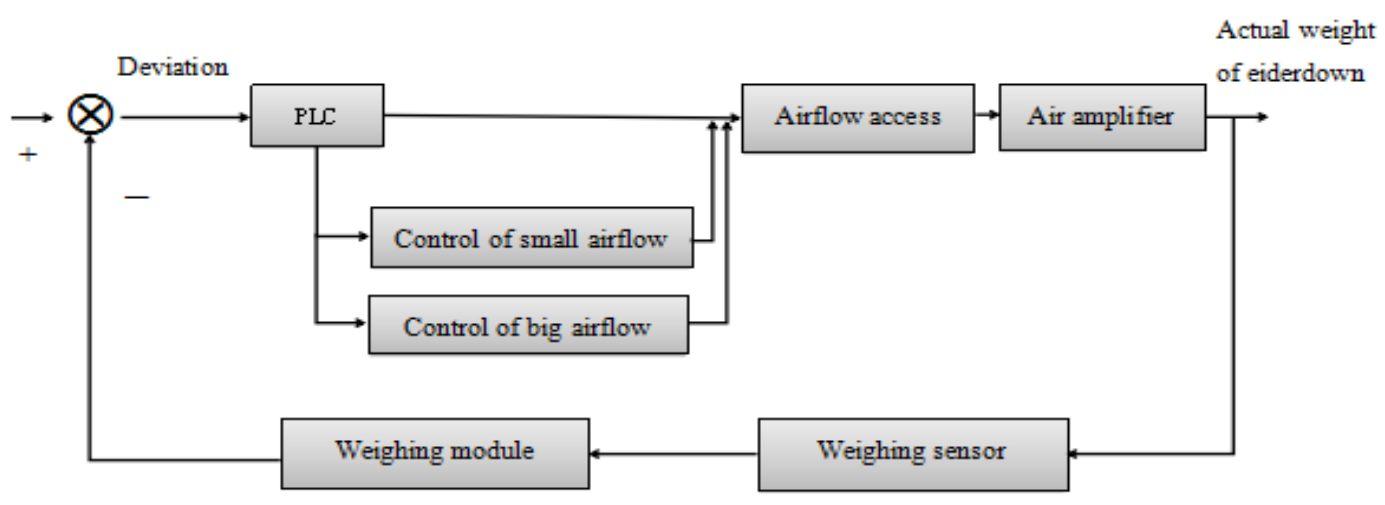

Fig.3 Control of weighing process

\section{Hardware of Control System}

This article adopts FATEK FBs-60MAT2-AC type PLC, 36 dot DC24 input ( 8 dot 20KHz, 8 dot sum $5 \mathrm{KHz}$ ), 24 dot input ( 8 dot 20KHz), 1 communication pot (open-ended 3 ). The weighing module adopts FBs-2LC, FBs-2LC is the analog input module of FATEK-FBs type PLC and used for measuring the weak signal of weighing sensor. This module shows the measuring signal with 16 directed numbers, and it is also equipped with sampling signal averaging and automatic zero compensation function to filter the on-site noise. The weighing module is tandem connected to the PLC extended interface to complete the 24VDC and input wiring. After execute FATEK PLC program software WinProladder, set load-cell metaplanning form start buffer, load-cell reading value start buffer and load-cell measuring start buffer in the LC module, then it can be directly read measure value in the buffer.

As the upper computer of the whole system, the touch screen can control the equipment operation 
and input controls parameter to monitor the operation. This system adopts FATEK UniCon system with touch screen for HU102E-00 type. The COMI port can be built communication with PLC through a communication wiring RS3223, and the USB port is used for upload and download of programs on the touch screen.

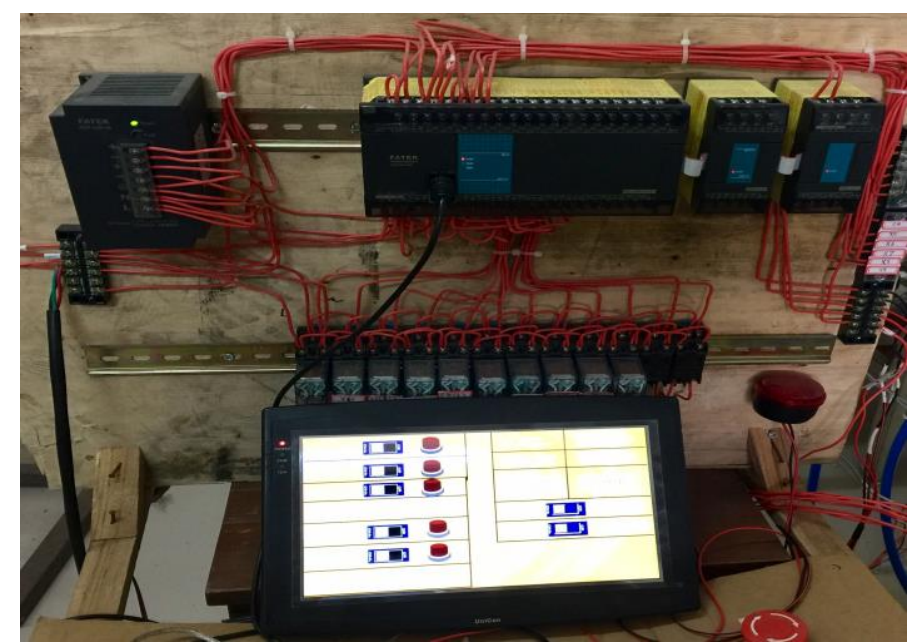

Fig.4 Experimental platform of PLC hardware

\section{Software Design of Control System}

\subsection{Design of PLC Program.}

In order to get more accurate weight value, it is required to make set and calibration before weighing. Firstly it is required to check whether the weighing sensor of setting R4016 parameter identification weighing module path for special intermediate relay M1924 is installed. Set the M0and M2 to correct the weighing sensor. After connect the weighing sensor and on the PLC on-line state, switch the "OFF" key to "ON" key of tare on the web watcher, set the original value for 0 , then switch it to "OFF". Later it is required to place $100 \mathrm{~g}$ weight on the weighing sensor and switch the "OFF" key to "ON" of correct. Set the weight value of full scales for 100, the switch to "OFF" key. After calibration, it can be started to weigh.

According to the requirements the I/O signals of distribution control system are shown in Figure 1. The pneumatic circuit diagram of control system and FBs-2LC wiring diagram of weighing module is respectively shown in Figure 5 and Figure 6.

Table $1 \mathrm{I} / \mathrm{O}$ address allocation

\begin{tabular}{|c|c|c|c|}
\hline \multicolumn{2}{|c|}{ Output signal } \\
\hline No. & Input & Output & Symbol \\
\hline 1 & Y0 & 1\# open material valve & KA1 \\
\hline 2 & Y1 & 1\# close material valve & KA2 \\
\hline 3 & Y2 & 1\# air flow circuit & KA3 \\
\hline 4 & Y3 & 1\# small air flow circuit & KA4 \\
\hline 5 & Y4 & 1\# flash buzzer & KA5 \\
\hline
\end{tabular}




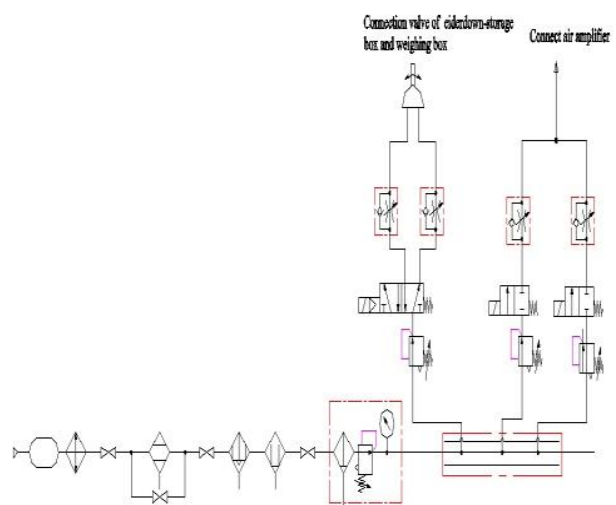

Fig.5 Design drawing for airflow weighing system

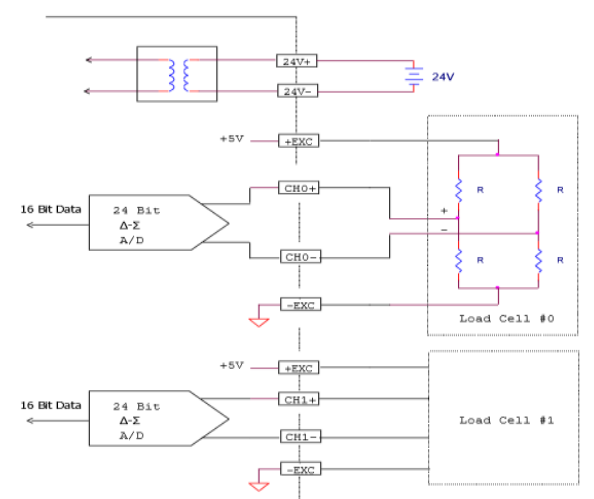

Fig.6 FBs-2LC wiring diagram circuit of of weighing module

\subsection{Main Interface Set for Touch Screen.}

This control system adopts touch screen to get communication with PLC and make parameter set and condition monitoring to the whole control system, the show picture of main interface is shown in Figure 7. This interface contains: master start, open the material valve, close the material valve, time of big airflow, quick charging, slow charging, time of small airflow, set weight of eiderdown, actual weight of eiderdown, tare and calibration. It is convenient for operation, reading the value of time or amending parameter of weight value.

Before weighting, it is required to make tare and checking on the touch screen to realize the precious weighing. On the set of touch screen it is available to make set the weight of eiderdown needed to be weighted by hand.

The displays of all the values in the figure are respectively relevant with the reservoir in PLC, through FATEK ASCII protocol it can read the values to the corresponding reservoir of PLC and then show them on the interface. During the weighing, the needed time of eiderdown transporting for small airflow and big airflow is shown dynamically on the touch screen.

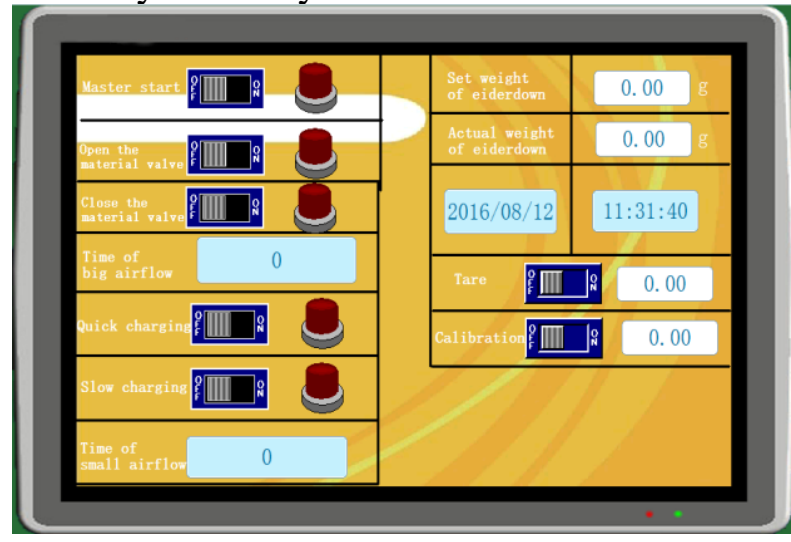

Fig. 7 Main interface of touch screen

\section{Experimental Process and Analysis of Result}

The set weight of eiderdown for this experiment is respectively for $80 \mathrm{~g}$ and $150 \mathrm{~g}$. It is required to set the values of big and small airflow pressure and set the required time for weighing process under the condition for reading the values of each air pressure on the touch screen. Then input the experimental data respectively to the MATLAB working space shown in the Figure 8, 10, 12 and 14 and use the fitting function of polynomial to fit the data. Input the following program in the order line, it will be obtained the matrix of multinomial coefficient. The fitting conditions are shown in Figure 9, 11,13 and 15 .

\section{0g:}


Table 3 Combination time for big airflow and small airflow

\begin{tabular}{ccccccccc}
\hline & 2.4 & 2.6 & 2.8 & 3.0 & 3.2 & 3.4 & 3.6 & 3.8 \\
0.4 & 180.27 & 169.06 & 159.30 & 144.15 & 131.83 & 118.12 & 107.46 & 96.28 \\
0.6 & 176.94 & 165.73 & 155.97 & 140.82 & 128.50 & 114.79 & 104.13 & 92.95 \\
0.8 & 172.97 & 161.76 & 152.00 & 136.85 & 124.53 & 110.82 & 100.16 & 88.98 \\
1.0 & 167.15 & 155.94 & 146.18 & 131.03 & 118.71 & 105.00 & 94.34 & 80.62 \\
1.2 & 164.61 & 153.40 & 143.64 & 128.49 & 116.17 & 102.46 & 91.80 & 80.62 \\
1.4 & 160.09 & 148.88 & 139.12 & 123.97 & 111.65 & 97.94 & 87.28 & 76.10 \\
1.6 & 154.56 & 143.35 & 133.59 & 118.44 & 106.12 & 92.41 & 81.75 & 70.57 \\
1.8 & 149.98 & 138.77 & 129.01 & 113.86 & 101.54 & 87.83 & 77.17 & 65.99 \\
\hline
\end{tabular}

150g:

Table 4 Combination time for big airflow and small airflow

\begin{tabular}{ccccccccccc}
\hline & 3.0 & 3.2 & 3.4 & 3.6 & 3.8 & 4.0 & 4.2 & 4.4 & 4.6 & 4.8 \\
0.8 & 359.42 & 336.96 & 314.9 & 289.17 & 260.32 & 234.46 & 212.32 & 191.21 & 179.18 & 162.08 \\
1.0 & 352 & 329.54 & 307.48 & 281.75 & 252.9 & 227.04 & 204.9 & 183.79 & 171.76 & 154.66 \\
1.2 & 344.91 & 322.45 & 300.39 & 274.66 & 245.81 & 219.95 & 197.81 & 176.7 & 164.67 & 147.57 \\
1.4 & 337.19 & 314.73 & 292.67 & 266.94 & 238.09 & 212.23 & 190.09 & 168.98 & 156.95 & 139.85 \\
1.6 & 329.26 & 306.8 & 284.74 & 259.01 & 230.16 & 204.3 & 182.16 & 161.05 & 149.02 & 131.92 \\
1.8 & 318.92 & 296.46 & 274.4 & 248.67 & 219.82 & 193.96 & 171.82 & 150.71 & 138.68 & 43.33 \\
2.0 & 307.1 & 284.64 & 262.58 & 236.85 & 208 & 182.14 & 160 & 138.89 & 126.86 & 109.76 \\
2.2 & 297.42 & 274.96 & 252.9 & 227.17 & 198.32 & 172.46 & 150.32 & 129.21 & 117.18 & 100.08 \\
2.4 & 288.88 & 266.42 & 244.36 & 218.63 & 189.78 & 163.92 & 141.78 & 120.67 & 108.64 & 91.54 \\
2.6 & 281.5 & 259.04 & 236.98 & 211.25 & 182.4 & 156.54 & 134.4 & 113.29 & 101.26 & 84.16 \\
\hline
\end{tabular}

It is known from the curve-fitting graph with the increasing of airflow value, the time decreases, and known from the combination time of big airflow and small airflow. When the combination time for $\left(4.8 \mathrm{~kg} / \mathrm{cm}^{2}, 2.6 \mathrm{~kg} / \mathrm{cm}^{2}\right)$ and $\left(3.8 \mathrm{~kg} / \mathrm{cm}^{2}, 1.8 \mathrm{~kg} / \mathrm{cm}^{2}\right)$ is respectively for 65.99 seconds and 84.16 seconds for the least. Therefore on the applicable scale of air pressure, the best value of big airflow and small airflow is respectively for: $80 \mathrm{~g}: 3.8 \mathrm{~kg} / \mathrm{cm}^{2}, 1.8 \mathrm{~kg} / \mathrm{cm}^{2} ; 150 \mathrm{~g}: 4.8 \mathrm{~kg} / \mathrm{cm}^{2}, 2.6 \mathrm{~kg} / \mathrm{cm}^{2}$.

\section{Conclusion}

This article adopts FBs-60MAT2-AC type PLC of Taiwan FATEK Co., Ltd. and HU102E-00 touch screen of PATEK series to design the quantitative delivery and dynamic weighing control system for eiderdown. It adopts PLC to control the high air pressure and low air pressure of two-path and adjust transporting speed piecewise. Combined with the reflected real-time weight data from weighing sensor, it can realize the goal of transporting eiderdown accurately and quantitatively. Through series of weighing experiments, it also adopts MATLAB to make least square fit for data and obtains the results that best big air pressure and small pressure under the different quantitative value to realize the quick and accuracy weighing.

\section{Acknowledgments}

Henan Province science and technology research project "Research on KeyTechnology of Automatic Filling Machine for Garment";Henan Xinya Clothing Co.,Ltd.topic"Design and development of semi-automatic filling machine".

\section{References}

[1]. Zhang Wei. weighing box[P].Chinese patent:CN203287088 U,2013-11-13:

[2]. Zhao Yanwei.Design and research on computer eiderdown-filling machine [J].Mechanical design, 1994, 06:43-46. 
[3]. Kong Zhen. Design and research on control system of eiderdown-filling machine based on PLC [D].Shangdong: Shandong University of Science and Technology, 2014:1-3

[4]. $\mathrm{Xu}$ Jianxin. Weighing box of eiderdown used for eiderdown-filling machine[P].Chinese patent:CN104655243 A,2015-05-27:

[5]. $\mathrm{Xu}$ Jianxin. Anti-static weighing box of eiderdown[P].Chinese patent:CN104677473 A,2015-06-03:

[6]. Liu Hangdong. Weighing box with accurate weighting[P].Chinese patent:CN203360671 U,2013-12-25:

[7]. Qian Weibo. Weighing box used for eiderdown-filling machine with buffering function[P].Chinese patent:CN204461569 U,2015-07-08:

[8]. Zhao Yanwei, Wang Wanliang. Real-time prediction and control on computer eiderdown-filling machine [J].Automation in engineering industry, Vol.23 (1994) No. 4, p. 263-256.

[9]. Wang Wanliang, Predication algorithm for orthogonal approximation and the application on computer eiderdown-filling machine [J].Information and control, 1994, 04:253-256.

[10]. Qiao QIAO, liang-zhi FAN.Control system design of automatic down-filling machine based on ARM7TDMI [J]. Hydromechatronics Engineering, Vol.42 (2014) No. 6, p. 74-77.

[11]. Qiao Qiao, Zhang Jiangang, Wu Xiaoguang. Design of automatic eiderdown-filling machine based on ARM7TDMI [J]. Automation and instrument, 2011, 05:35-38.

[12]. Wang Ke.Design and research on automatic eiderdown-filling machine based on PLC [J].Science and China, 2015, 12(311):7-10. 